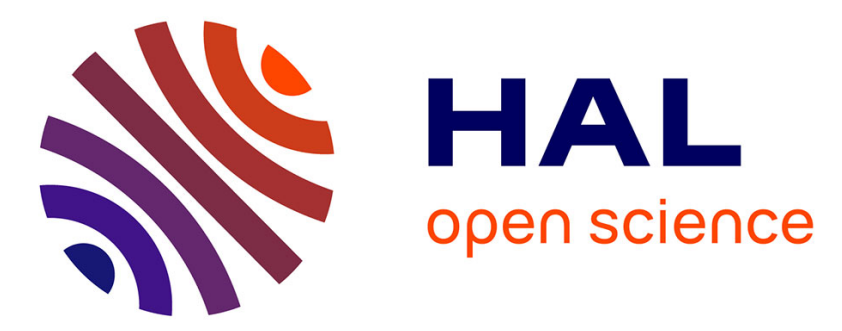

\title{
Hyper-Rayleigh Scattering as a New Chiroptical Method: Uncovering the Nonlinear Optical Activity of Aromatic Oligoamide Foldamers
}

Dominique Verreault, Kevin Moreno, Éric Merlet, Frédéric Adamietz, Brice Kauffmann, Yann Ferrand, Céline Olivier, Vincent Rodriguez

\section{To cite this version:}

Dominique Verreault, Kevin Moreno, Éric Merlet, Frédéric Adamietz, Brice Kauffmann, et al.. HyperRayleigh Scattering as a New Chiroptical Method: Uncovering the Nonlinear Optical Activity of Aromatic Oligoamide Foldamers. Journal of the American Chemical Society, 2019, 142 (1), pp.257263. 10.1021/jacs.9b09890 . hal-03007660

\section{HAL Id: hal-03007660 https://hal.science/hal-03007660}

Submitted on 24 Nov 2020

HAL is a multi-disciplinary open access archive for the deposit and dissemination of scientific research documents, whether they are published or not. The documents may come from teaching and research institutions in France or abroad, or from public or private research centers.
L'archive ouverte pluridisciplinaire HAL, est destinée au dépôt et à la diffusion de documents scientifiques de niveau recherche, publiés ou non, émanant des établissements d'enseignement et de recherche français ou étrangers, des laboratoires publics ou privés. 


\title{
Hyper-Rayleigh Scattering as a New Chiroptical Method: Uncovering the Nonlinear Optical Activity of Aromatic Oligoamide Foldamers
}

\author{
Dominique Verreault, ${ }^{*}{ }^{\dagger}$ Kevin Moreno, ${ }^{\dagger}$ Éric Merlet, ${ }^{\dagger}$ Frédéric Adamietz, ${ }^{\dagger}$ Brice Kauffmann, ${ }^{\S}$ Yann \\ Ferrand ${ }^{\ddagger}$ Céline Olivier, ${ }^{*}, \dagger$ and Vincent Rodriguez ${ }^{*}, \dagger$ \\ ${ }^{\dagger}$ Institut des Sciences Moléculaires, UMR 5255 CNRS, Université de Bordeaux, 351 Cours de la Libération, F-33405 Talence \\ Cedex, France \\ * Institut de Chimie et Biologie des Membranes et des Nano-objets, UMR 5248 CNRS, Université de Bordeaux, 2 rue Robert \\ Escarpit, F-33600 Pessac, France \\ $\S$ Institut Européen de Chimie et Biologie, Université de Bordeaux, UMS 3033 CNRS, 2 rue Robert Escarpit, F-33600 Pessac, \\ France
}

Supporting Information Placeholder

\begin{abstract}
Molecular helices based on self-organized aromatic oligoamide foldamers have been designed and prepared in their two enantiomeric forms in order to probe their second-order nonlinear chiroptical properties in solution. The quinoline oligoamides were rationally functionalized by electron-donating and electron-withdrawing groups to afford a gradual increase of the electronic polarization of the helical architectures. Their hyper-Rayleigh scattering (HRS) responses in solution were accordingly assessed, using either linearly polarized or circularly polarized incident light. Both methods allowed to observe nonlinear optical activity that was quantified, for the first time for molecular systems, through circular differential scattering intensity ratios. The hyper-Rayleigh optical activity (HROA) study reveals important charge transfer differences within the aromatic oligomers, depending on the helix handedness and on the extent of electronic polarization induced by the appended substituents. The origin of the enantiomeric difference is discussed considering both achiral and chiral contributions. Overall, we disclose the capabilities of HRS as a complementary chiroptical method, ideally suited for the analysis of chiral molecular and supramolecular systems in solution. Implementation of auxiliary polarization/detection techniques on our measurement setup is discussed to further increase the chiral discrimination sensitivity and extend the study to a wide rande of small molecular systems.
\end{abstract}

\section{- INTRODUCTION}

The determination of molecular chirality essentially relies on linear optical means that allow to quantify the optical activity of molecular and supramolecular systems. ${ }^{1,2}$ For instance, circular dichroism (CD) and, more recently circularly polarized luminescence (CPL), have become invaluable methods to probe chiroptical properties, respectively, from the ground state of nonracemic chiral chromophoric species, ${ }^{3-5}$ and from the excited states of emissive systems. ${ }^{6,7}$ Yet, a major drawback of linear chiroptical methods is their low chiral sensitivity due to weak chiral signals being dominated by the inherently large achiral background. ${ }^{6,8,9}$ For example, in CD and CPL, electric dipole interactions exceed by several orders of magnitude the mixed electric/magnetic dipole interactions at the origin of chiral contributions. ${ }^{10,11}$ Despite extensive studies with these methods, extracting the chiral signal remains a challenging task and has forced the development of various strategies, e.g., based on plasmonic substrates and nanostructures, ${ }^{12,13}$ novel polarization/detection schemes, ${ }^{14-17}$ and on the design/engineering of the chiral system itself. ${ }^{18-20}$

Notwithstanding, nonlinear optical (NLO) methods such as hyper-Rayleigh scattering (HRS) could offer an alternative approach and potentially overcome the limitation of linear chiroptical methods. HRS is an incoherent second-order nonlinear process by which two photons with the same frequency $\omega$ interact with a non-centrosymmetric molecule to create a single photon with twice the original frequency $2 \omega$ (Figure 1A). ${ }^{21}$ As an optical probing method, HRS affords molecular-level information about the electronic charge distribution (dipolar/octupolar) and the symmetry of organic molecular and supramolecular systems dispersed in solution. ${ }^{22-28}$ Furthermore, as theorized in 1979 by Andrews and Thirunamachandran, nonlinear scattering methods such as HRS should also enable to measure the optical activity of chiral molecules and chiral supramolecular assemblies depending on the handedness of the incident circularly polarized light. ${ }^{29}$ As in linear chiroptical methods, achiral contributions due to electric dipolar interactions are still expected to be dominant in HRS, with the key difference that the mixed electric and magnetic dipoles are more comparable in magnitude (differing by $1-2$ orders only), ${ }^{21}$ thus making chiral contributions significant. Still, there has been so far no experimental evidence demonstrating the chiroptical capabilities of these methods on molecular systems, although very recently hyper-Rayleigh scattering optical activity has been reported for silver nanohelices dispersed in water. ${ }^{30}$ Besides instrumental factors (i.e., low detection limits) another possible reason for this state-of-affairs has been the lack of chiral molecular systems with sufficiently high NLO response. The NLO response being directly related to the molecular first hyperpolarizability, much effort has been devoted in recent years to the design of molecules that enhance this parameter. One successful approach has been to exploit the helicity of phenylene oligomers substituted with donor/acceptor groups. ${ }^{31}$ Several helical $\pi$-conjugated systems have also been shown to exhibit enhanced HRS response due to their intrinsic electronic delocalization. ${ }^{32-36}$ However, there has been no report about a possible chiral effect on the NLO response of these systems mainly because of the difficulty in producing both enantiomeric forms. 
A.

Linearly Polarized Light (LPL)

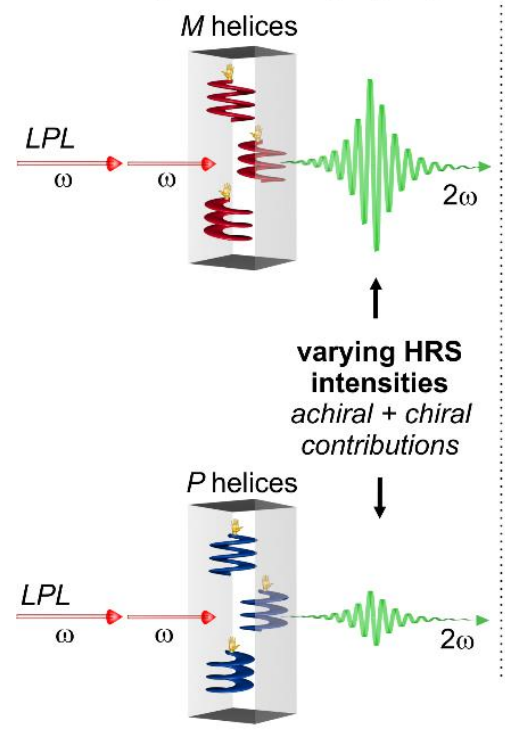

B. Circularly Polarized Light (CPL)

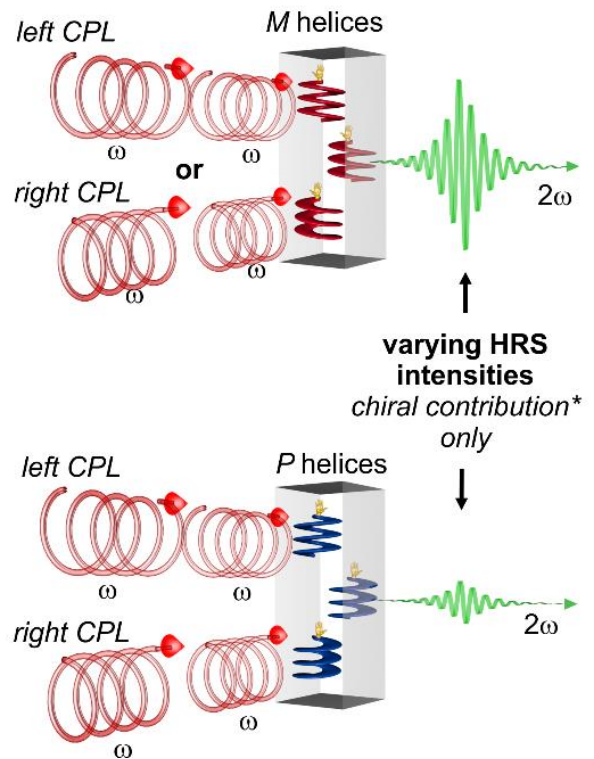

C.

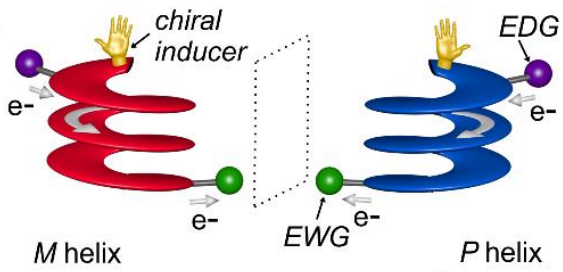

D.

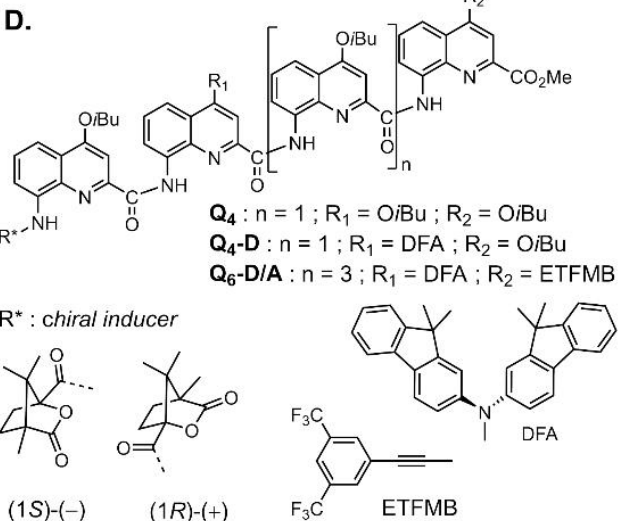

$(1 S)-(-)$ Figure 1. Schematic representation of non-racemic molecular systems in solution probed by hyper-Rayleigh scattering (HRS) using (A)
linearly polarized incident light (LP), and (B) circularly-polarized (CP) incident light, $\left({ }^{*}\right.$ for $\left.I_{2 \omega}^{\mathrm{RCP}}-I_{2 \omega}^{\mathrm{LCP}}\right)$. Incident light is obtained from laser excitation at $1064 \mathrm{~nm}$ (off-resonance) and detection is set at $532 \mathrm{~nm}$ (see ESI for experimental setup). (C) Cartoon representation of the induced polarization of non-racemic $M$ (red) and $P$ (blue) helices. The balls represent electron-donating groups (EDG, purple) and electronwithdrawing groups (EWG, green). The golden hand stands for the chiral inducer. The arrows represent the electron flow. (D) Chemical formulas of $\mathbf{Q}_{4}, \mathbf{Q}_{4}-\mathrm{D}$ and $\mathbf{Q}_{6}-\mathrm{D} / \mathbf{A}$; camphanic chiral inducers; electron-donating group, DFA, and electron-withdrawing group, ETFMB.

Among molecular helical structures, aromatic oligoamide foldamers are known to readily afford chiral self-organized architectures. These versatile synthetic oligomers adopt predictable and highly stable folded conformations in solution due to designed intramolecular interactions between adjacent units. ${ }^{37}$ In particular, oligomers of 8-amino-2-quinolinecarboxylic acid can give rise to helices with 2.5 units per turn and a pitch of $3.5 \AA .^{38}$ A complete preferential handedness can be induced through functionalization by a chiral inducer at the $\mathrm{N}$-terminus resulting in either pure righthanded $(P)$ or left-handed $(M)$ enantiomers. ${ }^{39}$ The synthetic flexibility of quinoline oligoamides is fostered by their sequential nature, providing highly modular structures that can be readily decorated by peripheral substituents with atomic precision. Moreover, aromatic oligoamides allow photoinduced charge transfer processes over long distances via multidimensional charge transport mechanisms ${ }^{40-42}$ and can also behave as strong CPL emitters. ${ }^{43,44}$

Here, we report the rational design and preparation of molecular helices based on a quinoline oligoamide backbone functionalized by electronically active substituents, along with the assessment of their NLO properties in solution. In particular, HRS studies were performed using either linearly-polarized (LP) incident light (Figure 1A) or circularly-polarized (CP) incident light (Figure 1B) to probe the enantiomeric differential scattering intensities and quantify, for the first time, the hyper-Rayleigh optical activity (HROA) of such chiral molecular systems. For the purpose of this study, a hexamer of 8-amino-2-quinolinecarboxylic acid was accordingly polarized through introduction of an electron-donor arylamine group at one end and of an electron-withdrawing group at the other. This design not only promotes charge injection from the appended donor substituent to the helix, but also provides a directionality of the electron flow towards the withdrawing substituent through the helix backbone (Figure 1C).

\section{- RESULTS AND DISCUSSION}

Optically pure tetramers and hexamers of quinoline were prepared and derivatized at the N-terminus by $(1 S)-(-)$ or $(1 R)-(+)$ camphanic chiral inducers to provide $P$ or $M$ helices, respectively (Figure 1D). ${ }^{38}$ In a general approach, the quinoline monomer bears a diverging isobutoxy side chain at position 4 of the aromatic ring, conferring a good solubility in organic media. First, tetramer $\mathbf{Q}_{4}$ was prepared to investigate the native polarization of the helix from the $\mathrm{N}$ - to the C-terminus. Then, in order to increase the electron density in the helix, tetramer $\mathbf{Q}_{4}-\mathbf{D}$ was functionalized at the position 2 of the sequence by insertion of the electron-rich group bis(9,9-dimethyl-9H-fluoren-2-yl)amine (DFA, Figure1D). To further increase the intramolecular electronic polarization, hexamer Q6-D/A was endowed with the same electron-donating group, but also with an electron-withdrawing group, 1-ethynyl-3,5bis(trifluoromethyl)benzene, at the position 6 of the sequence (ETFMB, Figure 1D). The synthesis of the oligomers is described in detail in the ESI. The handedness control for hexamers $P / M-\mathbf{Q}_{6-}$ D/A was assessed by ${ }^{1} \mathrm{H}$ NMR. As expected, the presence of a single set of sharp signals revealed a complete handedness induction by the camphanyl group. The single crystal X-ray structure of the racemate hexamer (i.e., without chiral inducer) corresponding to $\mathbf{Q}_{6}-\mathbf{D} / \mathbf{A}$ was obtained by slow diffusion of hexane into a solution of the oligomer in chloroform (ESI). The crystalline structure (Figure 2) revealed a canonical folding of the helix whereas the spatial localization of the functional groups was confirmed on opposite sides of the helix. 


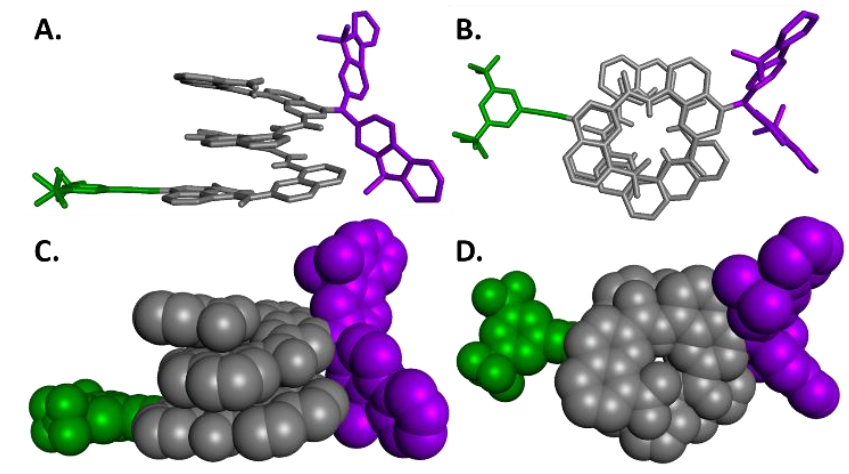

Figure 2. X-ray crystal structure of the $P$ helix of the racemate hexamer (i.e., without chiral inducer) corresponding to $\mathbf{Q}_{6}-\mathbf{D} / \mathbf{A}$. The helix and substituents are shown in stick representation: (A) side view, (B) top view; and in CPK representation: (C) side view, (D) top view. The non-polar hydrogens and isobutoxy side chains are omitted for clarity. The electron-donating group (DFA) is represented in purple and the electron-withdrawing group (ETFMB) in green.

Helical handedness of the oligomers was evidenced using electronic circular dichroism (ECD) absorption spectroscopy as shown in Figure 3. All the oligomers show intense absorption bands in the 230-430 nm range, characteristic of the quinoline chromophore skeleton. ${ }^{39,43,44}$ As expected, opposite signals are systematically observed for the three pairs of enantiomers. Based on previous reports on quinoline oligoamides, ${ }^{39,45}$ the positive sign of the bands around $400 \mathrm{~nm}$ for the enantiomers bearing the $(1 S)$ (-) camphanyl group indicates a $P$ handedness, while the negative sign of the bands observed for the enantiomer bearing the $(1 R)-(+)$ camphanyl group implies $M$ handedness. An additional absorption band is observed for $\mathbf{Q}_{4}-\mathbf{D}$ and $\mathbf{Q}_{6}-\mathbf{D} / \mathbf{A}$ in the visible region around $440 \mathrm{~nm}$, corresponding to induced CD absorption of the DFA substituent. ${ }^{44}$ It is worth noting that the ECD spectra show multiple Cotton effects, especially when substituents are present on the helix. The chiroptical activity difference in the absorption region of the quinolines thus indicates a perturbation of the electronic states of the helix scaffold upon functionalization.

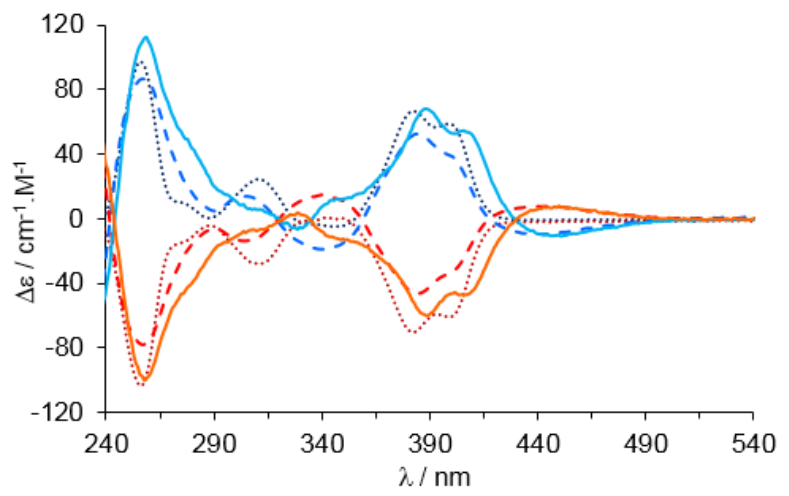

Figure 3. ECD spectra of $P-\mathbf{Q}_{4}$ (navy blue, dotted line), $M-\mathbf{Q}_{4}$ (burgundy, dotted line), $P-\mathbf{Q}_{4}-\mathbf{D}$ (blue, dashed line), $M-\mathbf{Q}_{4}-\mathbf{D}$ (red, dashed line), $P$-Q6-D/A (cyan, solid line) and $M-\mathbf{Q}_{6}-\mathbf{D} / \mathbf{A}$ (orange, solid line) recorded in $\mathrm{CHCl}_{3}(\mathrm{C}=100 \mu \mathrm{M}, 298 \mathrm{~K})$.

To assess the NLO properties of the quinoline oligomers, the HRS response of $\mathbf{Q}_{\mathbf{4}}, \mathbf{Q}_{\mathbf{4}}-\mathbf{D}$ and $\mathbf{Q}_{6}-\mathbf{D} / \mathbf{A}$ dissolved in chloroform was measured using LP incident light (Figure 1A) with laser excitation at $1064 \mathrm{~nm}$ (off-resonance) and detection at $532 \mathrm{~nm}$, following a procedure described previously. ${ }^{46}$ The monomer $\mathbf{Q}$ (ESI) was also tested for comparison. The effective first hyperpolarizability ( $\beta_{\mathrm{HRS}}$ ) and the depolarization ratio (DR), which are indicators of the HRS response, have been calculated assuming a simplified process invoking only dipolar electric transitions (see ESI). The corresponding data are gathered in Table 1. Overall, the oligomers show remarkable NLO response, with average enantiomeric $\left\langle\beta_{\mathrm{HRS}}\right\rangle$ values ranging from 518 at. u. $\left(\mathbf{Q}_{4}\right)$ to 9550 at. u. $\left(\mathbf{Q}_{6}-\mathbf{D} / \mathbf{A}\right)$. The tetramer $\mathbf{Q}_{4}$, devoid of electron-donor substituents, yields $\left\langle\beta_{\mathrm{HRS}}\right\rangle=518$ at. u., which is about two orders of magnitude larger than the response from hexahelicenes, ${ }^{32}$ but comparable to that predicted for pyridine-pyrimidine oligomers. ${ }^{35}$ Here, the whole helical structure likely acts as a charge reservoir over which a significant charge transfer occurs since the $\beta_{\text {HRS }}$ of $\mathbf{Q}_{4}$ proved to be about four times larger than the response resulting from the simple addition of four $\mathbf{Q}$ monomers ( 144 at. u.). Interestingly, the introduction of electron-donor $\left(\mathbf{Q}_{4}-\mathbf{D}\right)$ and donor/acceptor substituents $\left(\mathbf{Q}_{6}-\mathbf{D} / \mathbf{A}\right)$ on the helix backbone induces a significant increase of $\left\langle\beta_{\mathrm{HRS}}\right\rangle$ due to cooperative charge transfers within the helix. Besides, average DR values indicate that $\mathbf{Q}_{4}$ presents a near-octupolar response with $\langle\mathrm{DR}\rangle=2.17$, since for purely octupolar NLO chromophores such as crystal violet DR = 1.5. ${ }^{47,48}$ For $\mathbf{Q}_{4}-\mathbf{D}(\langle\mathrm{DR}\rangle=2.99)$ the introduction of an electrondonor group induces a stronger response towards dipolar $\pi$ conjugated 1D push-pull systems (for which $\mathrm{DR}=5$ ). As one could expect, this behaviour is even more pronounced for $\mathbf{Q}_{6}-\mathbf{D} / \mathbf{A}(\langle\mathrm{DR}\rangle$ $=3.54)$, where the helix is polarized by both donor and acceptor groups.

More strikingly, these standard linearly polarized HRS measurements tend to show that the NLO response of the foldamers depends on the helix handedness (Table 1). In particular, one can note that both $\beta_{\mathrm{HRS}}$ and DR values are different for each enantiomer, the enantiomeric difference increasing when adding electron-donor $\left(\mathbf{Q}_{4}-\mathbf{D}\right)$ and donor-acceptor groups $\left(\mathbf{Q}_{6}-\mathbf{D} / \mathbf{A}\right)$ (Figure 4). This highlights the effectiveness of HRS to unravel enantiomeric (hyper)polarizability differences in molecular systems.

To quantify the overall enantiomeric difference in the NLO response, one can define the following normalized ratio:

$$
\frac{\delta \beta_{\mathrm{HRS}}}{\left\langle\beta_{\mathrm{HRS}}\right\rangle}=\frac{\beta_{\mathrm{HRS}}^{(P)}-\beta_{\mathrm{HRS}}^{(M)}}{\frac{1}{2}\left(\beta_{\mathrm{HRS}}^{(P)}+\beta_{\mathrm{HRS}}^{(M)}\right)}
$$

Implicitly, this ratio includes achiral and chiral (purely dipolar electric) contributions, but may also include chiral and achiral (mixed dipolar electric/magnetic and dipolar/quadrupolar electric) contributions which correspond essentially to gyrotropic crossproduct terms (vide infra). The corresponding ratios, calculated according to eq 1 , are listed in Table 1 . Considering the absolute values, $\mathbf{Q}_{4}$ exhibits a relatively small enantiomeric difference (8\%) compared to Q4-D (20\%) and Q6-D/A (39\%) for which the enantiomeric difference increases with the degree of polarization induced by the substituents. In addition, $\mathbf{Q}_{4}-\mathbf{D}$ and $\mathbf{Q}_{6}$-D/A exhibit opposite signs of enantiomeric difference, which tends to show that antagonistic effects occur within these foldamers, depending on the helix handedness and the degree of polarization. 
Table 1. NLO parameters deduced from HRS measurements for quinoline monomer $\mathbf{Q}$, tetramers $\mathbf{Q}_{\mathbf{4}}, \mathbf{Q}_{\mathbf{4}}-\mathbf{D}$ and hexamer $\mathbf{Q}_{6}-\mathbf{D} / \mathbf{A}^{a}$

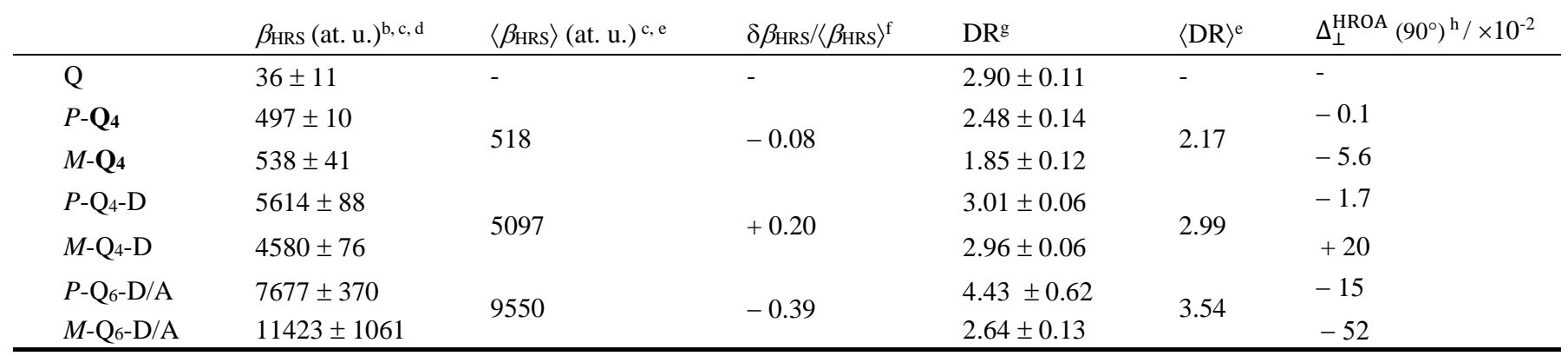

${ }^{a}$ Measurements in $\mathrm{CHCl}_{3}(298 \mathrm{~K}) ; \lambda_{\text {excitation }}=1064 \mathrm{~nm} ; \lambda_{\text {detection }}=532 \mathrm{~nm} .{ }^{b}$ Effective first hyperpolarizability. ${ }^{c}$ Using the $T$-convention: ${ }^{49} 1$ atomic unit (at. u.) $=3.621 \times 10^{-42} \mathrm{~m}^{4} \mathrm{~V}^{-1}=8.639 \times 10^{-33}$ esu. ${ }^{d}$ The reported uncertainties correspond to errors due to the fitting procedures. ${ }^{e}$ Average enantiomeric values. ${ }^{f}$ Enantiomeric difference ratio from eq $1 .{ }^{g}$ Depolarization ratio. ${ }^{h}$ Estimated circular differential scattering intensity ratio from eq $2\left(\mathrm{C}=10^{-3}\right.$

It is worth noting that these important enantiomeric differences represent the difference of charge transfer but without any chiral/achiral distinction. Hence, charge transfers can be achiral (1D), such as in $\pi$-conjugated push-pull systems, and/or axial (induced asymmetry) with a $2 \mathrm{D}$ (circular) or 3D (screw-like) character. Here, it is very likely that an axial charge transfer strongly contributes to the optical activity of the molecular helices.

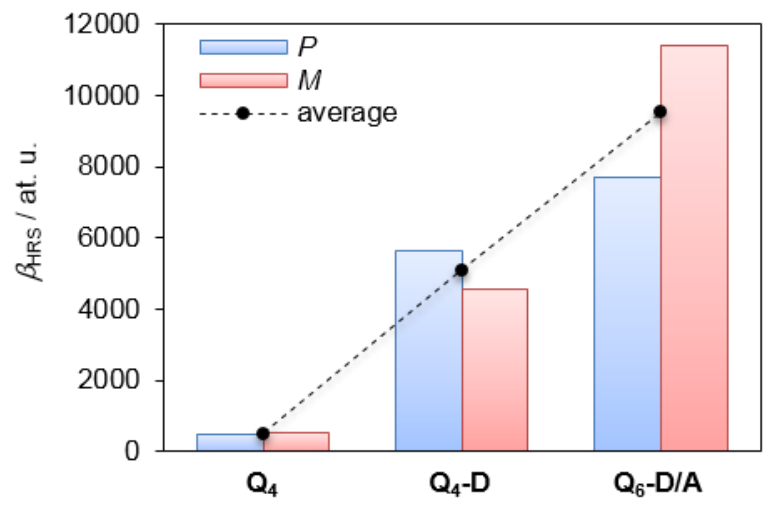

Figure 4. Effective first hyperpolarizability $\left(\beta_{\mathrm{HRS}}\right)$ of the $P$ (blue) and $M$ (red) enantiomers of $\mathbf{Q}_{\mathbf{4}}, \mathbf{Q}_{\mathbf{4}}-\mathbf{D}$ and $\mathbf{Q}_{6}-\mathbf{D} / \mathbf{A}$. Average enantiomeric values $\left\langle\beta_{\mathrm{HRS}}\right\rangle$ are represented as black dots.

To get deeper insight in the NLO activity of the oligomers, the HRS response of $P$ and $M$ enantiomers of $\mathbf{Q}_{4}, \mathbf{Q}_{4}-\mathbf{D}$ and $\mathbf{Q}_{6}-\mathbf{D} / \mathbf{A}$ has been also evaluated with static polarization measurements using both right-handed circularly-polarized (RCP) and left-handed circularly-polarized (LCP) incident light (Figure 1B). In standard linear chiroptical methods, a dimensionless differential ratio is usually introduced to quantify the corresponding optical activity, as for example, the circular intensity difference (CID) for vibrational Raman optical activity (VROA) $)^{50}$ or Kuhn's dissymmetry factor $\left(g_{\text {abs }}\right)$ for ECD. ${ }^{51}$ Similarly, for HROA, a circular differential scattering intensity ratio has been introduced and defined as: ${ }^{29}$

$$
\Delta_{\perp}^{\mathrm{HROA}}\left(90^{\circ}\right)=\frac{I_{2 \omega}^{\mathrm{RCP}}-I_{2 \omega}^{\mathrm{LCP}}}{\frac{1}{2}\left(I_{2 \omega}^{\mathrm{RCP}}+I_{2 \omega}^{\mathrm{LCP}}\right)}
$$

where $I_{2 \omega}^{\mathrm{RCP}}$ and $I_{2 \omega}^{\mathrm{LCP}}$ are the intensities of scattered secondharmonic light measured upon irradiation with RCP and LCP incident light, respectively. In eq 2 , the scattering geometry is set at the right angle $\left(90^{\circ}\right)$ and the $\mathrm{SH}$ intensity is analyzed vertically, i.e., perpendicularly to the scattering plane. It was shown that in this configuration the $\Delta_{\perp}^{\mathrm{HROA}}$ ratio (see eq S9 in ESI) contains exclusively normalized chiral (mixed dipolar electric/magnetic and dipolar/quadrupolar electric) absorption contributions. ${ }^{29}$

The corresponding $\Delta_{\perp}^{\mathrm{HROA}}$ ratios calculated from eq 2 are reported in Table 1 and summarized in Figure 5. It is important to mention that these ratios are only preliminary estimations as the calculation of eq 2 for binary solutions involves four separate measurements (two each for the solute and solvent), each with its own experimental uncertainty. Note first that, whatever the sign, the range of the differential ratios is within $\sim 1-10^{-2}$, which represents quite important chiroptical responses for molecular systems and demonstrates the high chiral sensitivity of HRS. Second, it appears that the enantiomers responses are not "mirror images" of each other, i.e., with the same amplitude but opposite signs, as it is typically the case with the above-mentioned linear chiroptical methods. Interestingly, the $\Delta_{\perp}^{\mathrm{HROA}}$ ratio of the $M$ enantiomers shows the same sign as the enantiomeric difference $\delta \beta_{\text {HRS }} /\left\langle\beta_{\text {HRS }}\right\rangle$ obtained from linearly polarized HRS measurements (see Table 1), and concomitantly, the $M$ enantiomers systematically show the closest octupolar-like DR. This seems to indicate that the $M$ helices are mostly dominated by chiral induced asymmetry, unlike the $P$ helices.

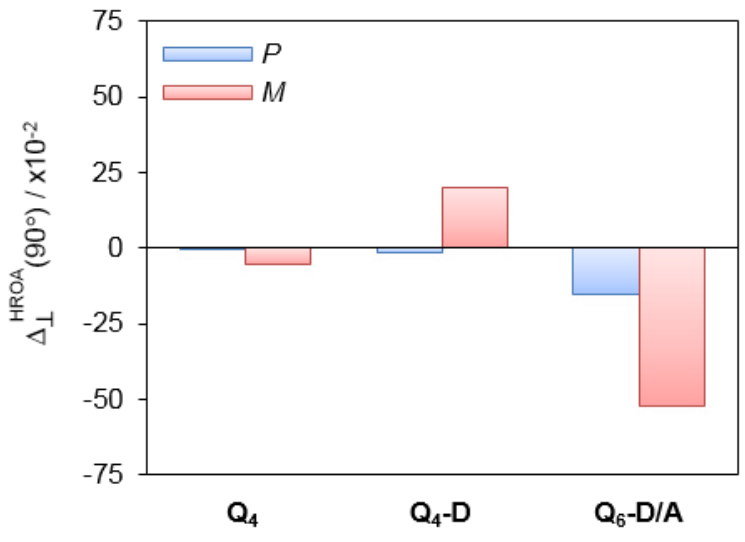

Figure 5. Estimated circular differential scattering intensity ratios of the $P$ (blue) and $M$ (red) enantiomers of $\mathbf{Q}_{4}, \mathbf{Q}_{\mathbf{4}}-\mathbf{D}$ and $\mathbf{Q}_{6}-\mathbf{D} / \mathbf{A}$ $\left(\mathrm{C}=10^{-3} \mathrm{M}\right)($ see ESI).

The reason for this asymmetric behavior originates from the fact that the first hyperpolarizability is a third-rank tensor. In short, the HROA response contains at least gyrotropic $\beta-J$ cross-product terms, ${ }^{29}$ where both $\beta$ and $J$ contain asymmetric Cartesian 
components $(i j k)$, in relation with the three involved optical fields, which may differ in amplitude and sign from one enantiomer to the other (Figure 5). In particular, the $\beta_{i j j}$ and $\beta_{i i j}$ components are likely to play an important role in the asymmetric response and may interfere in the isotropic averages probably leading to additive contributions for the $M$ enantiomers and opposing contributions for the $P$ enantiomers. Moreover, in the case of $\mathbf{Q}_{4}-\mathbf{D}$ and $\mathbf{Q}_{6}-\mathbf{D} / \mathbf{A}$ the appended substituents (although achiral) are susceptible to adopt different orientations, and thus the number of rotamers may also affect the overall differential scattering ratio.

\section{- CONCLUSION}

In conclusion, chiral molecular helices based on self-organized aromatic oligoamide foldamers have been specifically designed and prepared to probe their second-order NLO properties in solution. For this purpose, quinoline oligoamides were functionalized by electron-donor and electron-acceptor groups that gradually increase the electron density and charge delocalization over the helical backbone. HRS studies, performed with either LP or CP incident light allowed to observe differential scattering intensities depending on the helix handedness and to quantify, for the first time, the HROA of chiral molecular systems in solution. In particular, important charge transfer differences were observed upon rational introduction of electronically active substituents on the oligomeric structures. We postulate that this behavior originates from the nature of the charge transfers that can be achiral (1D, push-pull) and/or axial (2D, circular or 3D, helical) due to induced asymmetry. This asymmetry in the charge transfers has a significant impact on the isotropic averages leading to either additive or opposing contributions depending on the helix handedness and extent of electronic delocalization. Overall, this study highlights the capabilities of HRS as a complementary chiroptical method, ideally suited for the discrimination of chiral molecular and supramolecular systems in solution..

It is worth noting that additional polarization and detection schemes successfully developed for linear spectroscopic and chiroptical methods could also be applied to HRS to further improve the gain in chiral sensitivity. For instance, to eliminate fluctuation from both the laser source and the detection unit as well as solvent contributions (e.g., foldamer solutions), one can envision to implement polarization modulation between RCP and LCP incident light for dynamic measurements (rather than static measurements as in the current work), thus increasing the signalto-noise ratio by about 2-3 orders of magnitude. Setup optimization is currently under way to implement this technique. In addition, polarization modulation opens up the possibility for inline, time-resolved studies of chiral systems where dynamic measurements could be done, for example, on pure chiral liquids and solutions where the concentration of a given chiral molecule is progressively changed. Finally, the fact that the HRS response stems from a very small interaction volume (typically in the range of 10-100 pL) further enables to design and miniaturize experiments using smaller sampling volumes. This represents a major advantage of HRS as the preparation of chiral compounds, even in small quantities (i.e., of the order of the milligram), can be cost- and/or timeintensive.

\section{- EXPERIMENTAL SECTION}

A description of the synthesis and characterization of all new compounds, detailed reaction schemes, crystallographic data, experimental details of HRS measurements and instrumentation utilized in this study may be found in the Supporting Information.
- ASSOCIATED CONTENT

\section{Supporting Information}

The Supporting Information is available free of charge on the ACS Publications website at DOI:

Materials and methods; Synthetic procedures and characterization; Crystallographic data; HRS details.

\section{- AUTHOR INFORMATION}

\section{Corresponding Authors \\ * dominique.verreault@u-bordeaux.fr \\ * celine.olivier@u-bordeaux.fr \\ * vincent.rodriguez@u-bordeaux.fr}

ORCID

Dominique Verreault: 0000-0002-2487-3565

Yann Ferrand: 0000-0002-6552-6914

Céline Olivier: 0000-0002-3164-8403

Vincent Rodriguez: 0000-0001-6804-9757

\section{Notes}

The authors declare no competing financial interests.

\section{ACKNOWLEDGMENTS}

This research has been carried out with financial support from the French National Research Agency (ANR) in the framework of the "Investments for the Future" Programme IdEx Bordeaux LAPHIA (ANR-10-IDEX-03-02) and in the framework of the Laboratoire International Associé (LIA) - "Chiral Nanostructures for Photonic Applications" (CNPA, Japan). VR thanks the Région Nouvelle Aquitaine for financial support (CRA: 2015-1R10205-00004858).

\section{REFERENCES}

(1) Berova, N.; Polavarapu, P. L.; Nakanishi, K.; Woody, R. W. (Eds.) Comprehensive Chiroptical Spectroscopy: Applications in Stereochemical Analysis of Synthetic Compounds, Natural Products, and Biomolecules, Vol. 2, John Wiley \& Sons, Hoboken, NJ, 2012.

(2) Polavarapu, P. L. Renaissance in Chiroptical Spectroscopic Methods for Molecular Structure Determination. Chem. Rec. 2007, 7, 125-136.

(3) Berova, N.; Di Bari, L.; Pescitelli, G. Application of Electronic Circular Dichroism in Configurational and Conformational Analysis of Organic Compounds. Chem. Soc. Rev. 2007, 36, 914-931.

(4) Gottarelli, G.; Lena, S.; Masiero, S.; Pieraccini, S.; Spada, G. P. The Use of Circular Dichroism Spectroscopy for Studying the Chiral Molecular Self-Assembly: An Overview. Chirality 2008, 20, 471-485.

(5) Pescitelli, G.; Di Bari, L.; Berova, N. Application of Electronic Circular Dichroism in the Study of Supramolecular Systems. Chem. Soc. Rev. 2014, 43, 5211-5233.

(6) Sánchez-Carnerero, E. M.; Agarrabeitia, A. R.; Moreno, F.; Maroto, B. L.; Muller, G.; Ortiz, M. J.; de la Moya, S. Circularly Polarized Luminescence from Simple Organic Molecules. Chem. Eur. J. 2015, 21, 13488-13500.

(7) Longhi, G.; Castiglioni, E.; Koshoubu, J.; Mazzeo, G.; Abbate, S. Circularly Polarized Luminescence: A Review of Experimental and Theoretical Aspects. Chirality 2016, 28, 696-707.

(8) Barron, L. D.; Buckingham, A D. Vibrational Optical Activity. Chem. Phys. Lett. 2010, 492, 199-213.

(9) Berova, N.; Polavarapu, P. L.; Nakanishi, K.; Woody, R. W. (Eds.) Comprehensive Chiroptical Spectroscopy : Instrumentation, Methodologies, and Theoretical Simulations, Vol. 1, John Wiley \& Sons, Hoboken, NJ, 2012.

(10) Schellman, J. A. Circular Dichroism and Optical Rotation. Chem. Rev. 1975, 75, 323-331.

(11) Riehl, J P.; Richardson, F. S. Circularly Polarized Luminescence Spectroscopy. Chem. Rev. 1986, 86, 1-16. 
(12) Maoz, B. M.; Chaikin, Y.; Tesler, A. B.; Elli, O. B.; Fan, Z.; Govorov, A. O.; Markovich, G. Amplification of Chiroptical Activity of Chiral Biomolecules by Surface Plasmons. Nano Lett. 2013, 13, 1203-1209.

(13) Mohammadi, E.; Tsakmakidis, K. L.; Askarpour, A. N.; Dehkhoda, P.; Tavakoli, P.; Altug, H. Nanophotonic Platforms for Enhanced Chiral Sensing. ACS Photonics 2018, 5, 2669-2675.

(14) Helbing, J.; Bonmarin, M. Vibrational Circular Dichroism Signal Enhancement using Self-Heterodyning with Elliptically Polarized Laser Pulses. J. Chem. Phys. 2009, 131, 174507.

(15) Rhee, H.; Choi, J. S.; Starling, D. J.; Howell, J. C.; Cho, M Amplifications in Chiroptical Spectroscopy, Optical Enantioselectivity, and Weak Value Measurement. Chem. Sci. 2013, 4, 4107-4114.

(16) Rhee, H.; Eom, I.; Ahn, S.-H.;1 Song, K.-H.; Cho, M. Chiroptical Signal Enhancement in Quasi-Null-Polarization-Detection Geometry: Intrinsic Limitations. Phys. Rev. A 2015, 91, 053839.

(17) Begzjav, T. K.; Zhang, Z.; Scully, M. O.; Agarwal, G. S. Enhanced Signals from Chiral Molecules via Molecular Coherence. Opt. Expr. 2019 27, 13965-13977.

(18) Kumar, J.; Nakashima, T.; Kawai, T. Circularly Polarized Luminescence in Chiral Molecules and Supramolecular Assemblies. J. Phys. Chem. Lett. 2015, 6, 3445-3452.

(19) Han, J.; Yang, D.; Jin, X.; Jiang, Y.; Liu, M.; Duan, P. Enhanced Circularly Polarized Luminescence in Emissive Charge-Transfer Complexes. Angew. Chem. Int. Ed. 2019, 58, 7013-7019.

(20) Ma, K.; Chen, W.; Jiao, T.; Jin, X.; Sang, Y.; Yang, D.; Zhou, J.; Liu, M.; Duan, P. Boosting the Circularly Polarized Luminescence of Small Organic Molecules via Multi-Dimensional Morphology Control. Chem. Sci. 2019, 10, 6821-6827.

(21) Verbiest, T.; Clays, K.; Rodriguez, V. Second-Order Nonlinear Optical Characterization Techniques: An Introduction. CRC Press: Boca Raton, FL, 2009.

(22) Hendrickx, E.; Clays, K.; Persoons, A. Hyper-Rayleigh Scattering in Isotropic Solution. Acc. Chem. Res. 1998, 31, 675-683.

(23) Duncan, T. V.; Song, K.; Hung, S.-T.; Miloradovic, I.; Nayak, A.; Persoons, A.; Verbiest, T.; Therien, M. J.; Clays, K. Molecular Symmetry and Solution-Phase Structure Interrogated by Hyper-Rayleigh Depolarization Measurements: Elaborating Highly Hyperpolarizable $D_{2-}$ Symmetric Chromophores. Angew. Chem. Int. Ed. 2008, 47, 2978-2981.

(24) Asselberghs, I.; Flors, C.; Ferrighi, L.; Botek, E.; Champagne, B.; Mizuno, H.; Ando, R.; Miyawaki, A.; Hofkens, J.; Van der Auweraer, M. Clays, K. Second-Harmonic Generation in GFP-like Proteins. J. Am. Chem. Soc. 2008, 130, 15713-15719.

(25) Duboisset, J.; Matar, G.; Russier-Antoine, I.; Benichou, E.; Bachelier, G.; Jonin, C.; Ficheux, D.; Besson, F.; Brevet, P.-F. First Hyperpolarizability of the Natural Aromatic Amino Acids Tryptophan, Tyrosine, and Phenylalanine and the Tripeptide Lysine-Tryptophan-Lysine Determined by Hyper-Rayleigh Scattering. $J$. Phys. Chem. B 2010, 114, 13861-13865.

(26) Pandey, R.; Ghosh, S.; Mukhopadhyay, S.; Ramasesha, S.; Das. P. K. Geometry and Quadratic Nonlinearity of Charge Transfer Complexes in Solution using Depolarized Hyper-Rayleigh Scattering. J. Chem. Phys. 2011, 134, 044533.

(27) Castet, F.; Rodriguez, V.; Pozzo, J.-L.; Ducasse, L.; Plaquet, A.; Champagne, B. Design and Characterization of Molecular Nonlinear Optical Switches. Acc. Chem. Res. 2013, 46, 2656-2665.

(28) Nayak, A.; Park, J.; De Mey, K.; Hu, X.; Duncan, T. V.; Beratan, D. N.; Clays, K.; Therien, M. J. Large Hyperpolarizabilities at Telecommunication-Relevant Wavelengths in Donor-Acceptor-Donor Nonlinear Optical Chromophores. ACS Centr. Sci. 2016, 2, 954-966.

(29) Andrews, D. L.; Thirunamachandran, T. L. Hyper-Raman Scattering by Chiral Molecules. J. Chem. Phys. 1979, 70, 1027-1030.

(30) Collins, J. T.; Rusimova, K. R.; Hooper, D. C.; Jeong, H.-H.; Ohnoutek, L.; Pradaux-Caggiano, F.; Verbiest, T.; Carbery, D. R.; Fischer, P.; Valev, V. K., First Observation of Optical Activity in Hyper-Rayleigh Scattering. Phys. Rev. X 2019, 9, 011024.

(31) Panda, M.; Chandrasekhar, J. Exploiting the Helical Motif for Enhanced Nonlinear Optical Response: Hyperpolarizability of Substituted m-Phenylene Oligomers. J. Am. Chem. Soc. 1998, 120, 13517-13518.
(32) Botek, E.; Spassova, M.; Champagne, B.; Asselberghs, I.; Persoons, A.; Clays, K. Hyper-Rayleigh Scattering of Neutral and Charged Helicenes. Chem. Phys. Lett. 2005, 412, 274-279.

(33) Bossi, A.; Licandro, E.; Maiorana, S.; Rigamonti, C.; Righetto, S.; Stephenson, G. R.; Spassova, M.; Botek, E.; Champagne, B. Theoretical and Experimental Investigation of Electric Field Induced Second Harmonic Generation in Tetrathia[7]helicenes. J. Phys. Chem. C 2008, 112, 7900 7907.

(34) Champagne, B.; Labidi, S. L. Second-Order Nonlinear Optical Responses of Heptahelicene and Heptathiahelicene Derivatives. Chem. Phys. Lett. 2016, 644, 195-200.

(35) Botek, E.; Castet, F.; Champagne, B. Theoretical Investigation of the Second-Order Nonlinear Optical Properties of Helical Pyridine-Pyrimidine Oligomers. Chem. Eur. J. 2006, 17, 8687-8695.

(36) Van Cleuvenbergen, S.; Asselberghs, I.; Vanormelingen, W.; Verbiest, T.; Franz, E.; Clays, K.; Kuzyk, M. G.; Koeckelberghs, G. Record-high Hyperpolarizabilities in Conjugated Polymers. J. Mater. Chem. C 2014, 2 4533-4538.

(37) Huc, I. Aromatic Oligoamide Foldamers. Eur. J. Org. Chem. 2004, 17

(38) Jiang, H.; Léger, J.-M.; Huc, I. Aromatic $\delta$-Peptides. J. Am. Chem. Soc 2003, 125, 3448-3449.

(39) Kendhale, A. M.; Poniman, L.; Dong, Z.; Laxmi-Reddy, K.; Kauffmann, B.; Ferrand, Y.; Huc, I. Absolute Control of Helical Handedness in Quinoline Oligoamides. J. Org. Chem. 2011, 76, 195-200. (40) Wolffs, M.; Delsuc, N.; Veldman, D.; Vân Anh, N.; Williams, R. M Meskers, S. C. J.; J. Janssen, R. A.; Huc, I.; Schenning, A. P. H. J. Helical Aromatic Oligoamide Foldamers as Organizational Scaffolds for Photoinduced Charge Transfer. J. Am. Chem. Soc. 2009, 131, 4819-4829. (41) Li, X.; Markandeya, N.; Jonusauskas, G.; McClenaghan, N. D.; Maurizot, V.; Denisov, S. A.; Huc, I. Photoinduced Electron Transfer and Hole Migration in Nanosized Helical Aromatic Oligoamide Foldamers. $J$. Am. Chem. Soc. 2016, 138, 13568-13578.

(42) Méndez-Ardoy, A.; Markandeya, N.; Li, X.; Tsai, Y.-T.; Pecastaings, G.; Buffeteau, T.; Maurizot, V.; Muccioli, L.; Castet, F.; Huc, I.; Bassani, D. M. Multi-dimensional Charge Transport in Supramolecular Helical Foldamer Assemblies. Chem. Sci. 2017, 8, 7251-7257.

(43) Zheng, D.; Zheng, L.; Yu, C.; Zhan, Y.; Wang, Y.; Jiang, H. Significant Enhancement of Circularly Polarized Luminescence Dissymmetry Factors in Quinoline Oligoamide Foldamers with Absolute Helicity. Org. Lett. 2019, 21, 2555-2559.

(44) Merlet, E.; Moreno, K.; Tron, A.; McClenaghan, N.; Kauffmann, B.; Ferrand, Y.; Olivier, C. Aromatic Oligoamide Foldamers as Versatile Scaffolds for Induced Circularly Polarized Luminescence at Adjustable Wavelengths. Chem. Commun. 2019, 55, 9825-9828.

(45) Dolain, C.; Jiang, H.; Léger, J.-M.; Guionneau, P.; Huc, I. Chiral Induction in Quinoline-Derived Oligoamide Foldamers: Assignment of Helical Handedness and Role of Steric Effects. J. Am. Chem. Soc. 2005, 127, 12943-12951.

(46) Rodriguez, V.; Grondin, J.; Adamietz, F.; Danten, Y. Local Structure in Ionic Liquids Investigated by Hyper-Rayleigh Scattering. J. Phys. Chem. $B$ 2010, 114, 15057-15065.

(47) Chui, T. W.; Wong, K. Y. Study of Hyper-Rayleigh Scattering and Two-Photon Absorption Induced Fluorescence from Crystal Violet. $J$. Chem. Phys. 1998, 109, 1391-1396.

(48) Castet, F.; Blanchard-Desce, M.; Adamietz, F.; Poronik, Y. M.; Gryko, D. T.; Rodriguez, V. Experimental and Theoretical Investigation of the First-Order Hyperpolarizability of Octupolar Merocyanine Dyes. ChemPhysChem 2014, 15, 2575-2581.

(49) Willetts, A.; Rice, J. E.; Burland, D. M.; Shelton, D. P. Problems in the Comparison of Theoretical and Experimental Hyperpolarizabilities. J. Chem. Phys. 1992, 97, 7590-7599.

(50) Barron, L. D.; Buckingham, A. D. Rayleigh and Raman Scattering from Optically Active Molecules. Mol. Phys. 1971, 20, 1111-1119.

(51) Kuhn, W. The Physical Significance of Optical Rotatory Power. Trans. Faraday Soc. 1930, 26, 293-308. 


\section{GRAPHICAL ABSTRACT}

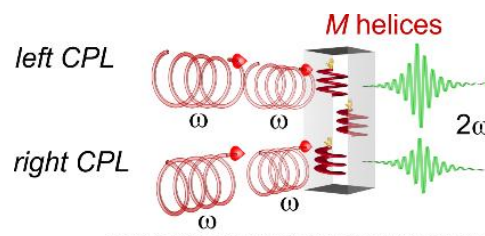

$H R S$ intensities

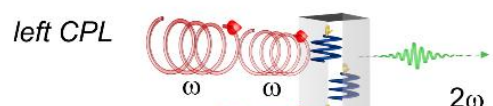

are $A L L$ different

right $C P L 0_{\omega}^{00} \underbrace{}_{P \text { helices }}{ }^{\circ}$ 